\title{
Use of hypnotics and the risk of or mortality from heart disease: a meta-analysis of observational studies
}

\author{
Young-Hyo Kim ${ }^{1}$, Hong-Bae Kim ${ }^{1,2}$, Do-Hyoung Kim ${ }^{1}$, Ja-Young Kim ${ }^{1}$, and Hyun-Young Shin ${ }^{1,2}$
}

${ }^{1}$ Department of Family Medicine, Myongji Hospital, Goyang; ${ }^{2}$ Department of Family Medicine, Yonsei University Graduate School of Medicine, Seoul, Korea

\author{
Received: August 31, 2016 \\ Revised : December 5, 2016 \\ Accepted: February 12, 2017

\section{Correspondence to} \\ Hong-Bae Kim, M.D. \\ Department of Family Medicine, \\ Myongji Hospital, 55 Hwasu-ro \\ 14beon-gil, Deokyang-gu, Goy- \\ ang 10475 , Korea \\ Tel: +82-31-810-5420 \\ Fax: +82-31-969-0500 \\ E-mail: hongbaig6@mjh.or.kr
}

\begin{abstract}
Background/Aims: Some observational epidemiologic studies have reported conflicting results on the relationship between hypnotics use and the risk of developing and/or dying from heart disease. We investigated these associations using a meta-analysis of available literatures.

Methods: We searched the databases PubMed and EMBASE, along with the bibliographies of relevant articles to find additional publications in February 2016.

Results: Of 495 articles satisfying our initial criteria, two case-control studies and six cohort studies met our inclusion criteria and were included in the final analyses. Compared with never having used any kind of hypnotics, the odds ratio for overall use was 0.84 for risk of or mortality from heart disease (95\% confidence interval, 0.79 to 0.89 ) in a random-effects meta-analysis of all eight studies. With respect to the geographical region, use of hypnotics was associated with a decreased risk or mortality of heart disease in Asia but not in Western countries. Among various types of sleep medications, zolpidem showed a decreased risk $(-29 \%)$ of developing or dying from heart disease, but benzodiazepines were related with an increased risk (80\%) of or mortality from heart disease.

Conclusions: The current meta-analysis of observational epidemiological studies suggested an evidence of association between hypnotics use and a decreased risk of heart disease.
\end{abstract}

Keywords: Hypnotics; Heart disease; Observational study; Meta-analysis

\section{INTRODUCTION}

Ailments of sleep have become one of the critical health problems giving detrimental effects on the quality of life of many people $[1,2]$. Almost $30 \%$ of people worldwide complaint more than one of sleep problems $[3,4]$. As a result, the current intake of hypnotics is estimated to generally range between $3 \%$ and $12 \%$, doubling in elderly people $[5,6]$.

Hypnotics have an alleviating effect on insomnia but can have several adverse effects. Sleeping pills can worsen daytime somnolence as well as memory performances [7]. For example, benzodiazepines (BZDs) have come to play a pivotal role for treating insomnia and are still widely prescribed to manage a sleep disturbance [8]. However, BZDs are also known to have some side effects, such as confusion, amnesia, and tremors. Zolpidem is a non-BZD hypnotic drug used for the management of insomnia [9] and can cause common adverse effects including headache, amnesia, and short-term memory loss [10].

With respect to the risk of heart disease due to hyp- 
notics, previous in vitro laboratory and animal studies have demonstrated a relationship between the use of hypnotics and increased risk of heart disease. Mailliet et al. [11] reported that diazepam injection could induce adverse effects on cardiovascular parameters such as blood pressure and heart rate in rats. Another in vitro cell study showed that overdose of zolpidem might be associated with the risk of acquired long QT syndrome [12]. For observational epidemiological studies, the relationship between hypnotics consumption and the risk of heart disease remains controversial. For example, in a 10-year follow-up research of 500 men [13], there was no significant link between use of hypnotics and the risk of cardiovascular disease. However, another observational study [14] found that use of BZD was associated with an increased risk of ischemic heart disease. In a cohort study in middle-aged people [15], regular use of hypnotics was also strongly associated with increased mortality from coronary artery disease.

To date, there have been no quantitative meta-analyses regarding this topic. In the current study, we investigated the associations between use of hypnotics and the incidence of heart disease by performing a metaanalysis of observational epidemiologic studies such as case-control studies and cohort studies.

\section{METHODS}

\section{Data sources}

We reviewed the PubMed, EMBASE databases to establish potentially relevant articles related to hypnotics and heart disease (in January 1971 through in February 2016). We also searched the bibliographies of related articles to find additional publications. Common keywords used were as follows: "hypnotics," "sleep medication," "sleeping pill," "BZD," "zolpidem," and "zopiclone," "cardiovascular," "heart," “coronary," and "valvular." There were no restrictions on the language of publication.

\section{Study selection}

We chose studies which reported the effect of hypnotics on the risk or mortality from heart disease. Our meta-analysis was restricted to observational epidemiologic studies such as case-control studies and cohort studies. Included studies should demonstrate outcome mea- sures with adjusted odds ratio (OR) or risk ratio (RR) and 95\% confidence interval (CI). If data were duplicated or shared between more than one study, the first published study was included in the analysis. We did not include unpublished studies and abstracts that were only presented at academic conferences or were not published in peer-reviewed journals.

Three authors (Y.H.K., H.Y.S., and H.B.K.) assessed the eligibility of all studies retrieved from the databases following the above selection criteria independently. If arguments occurred between evaluators, they were resolved by discussion.

\section{Assessment of methodological quality}

The methodological quality of the included studies was based on the Newcastle-Ottawa Scale (NOS) for assessing the quality of case-control studies and cohort studies in meta-analyses [16]. A star system of the NOS ranges from o to 9 and consists of three subscales: selection of studies, comparability, and exposure. We considered a study awarded stars of more than a mean score of each study type as a high-quality study since the criteria for the high- or low-quality of a study have not been established.

\section{Statistical analyses}

To calculate a pooled OR or RR with its $95 \%$ CI, we used the adjusted ORs or RRs and 95\% CIs in each study, which report the association between hypnotics use (highest use vs. never use) and heart disease. We examined the heterogeneity in the results across studies using the Higgins $I^{2}$, which measures the percentage of total variation across studies [17]. The $I^{2}$ was calculated as follows:

$$
I^{2}=100 \% \times(Q-d f) / Q,
$$

where $\mathrm{Q}$ is Cochran's heterogeneity statistic and df, the degrees of freedom. Negative values of $I^{2}$ were set to zero; the $I^{2}$ results are between o\% (no observed heterogeneity) and $100 \%$ (maximal heterogeneity) [17]. An $I^{2}$ value greater than $50 \%$ was considered to indicate substantial heterogeneity.

When substantial heterogeneity was not observed, the fixed-effects model was implemented using the Woolf's (inverse variance) method for the pooled estimate. When substantial heterogeneity was observed, the random-effects model was implemented using the DerSimonian 
and Laird method for the pooled estimate [17].

In the final analysis, we evaluated publication bias regarding the studies by using Begg's funnel plot and Egger's test. If publication bias existed, Begg's funnel plot would be asymmetrical or the $p$ value would be less than 0.05 by Egger's test. We used the Stata SE version 13.0 (Stata Corp., College Station, TX, USA) for the statistical analysis.

\section{RESULTS}

\section{Identification of relevant studies}

A total of 495 articles were obtained by assessing two databases and hand-searching relevant bibliographies. We excluded 134 duplicated articles and additional 341 articles that did not achieve the inclusion criteria. We reviewed the full texts of the remaining 20 articles. We excluded 12 articles among these articles because of the following reasons: not relevant to our analysis $(n=5)$, insufficient data $(n=1)$, using data from pre-existing heart disease $(n=4)$, and using an acute biomarker as exposure $(\mathrm{n}=2)$. The remaining eight studies (two case-control studies $[18,19]$ and six cohort studies $[15,20-24])$ were included in the final analysis (Fig. 1).

\section{Characteristics of studies included in the final analysis}

We identified a total of eight observational studies including 1,355,793 participants (8,689 patient cases and $1,347,104$ controls) [15,18-24]. In studies reporting age, the mean age was 56.2 years (range, 18 to 102). Table 1 demonstrates the general characteristics of the studies included in the final analysis. Based on the study design, two case-control studies ( $\mathrm{n}=918$ cases) $[18,19]$ and six prospective cohort studies ( $\mathrm{n}=7,771$ cases) $[15,20-24]$ were included. Only one study [19] collected hospital-based data. As for the types of heart disease, only one study [22] demonstrated the risk of heart failure hospitalization, and other seven studies investigated the risk of cardiovascular disease occurrences [15,18-21,23,24]. Two studies examined the risk of heart diseases [19,22], and six studies reported the mortality from heart disease $[15,18,20,21,23,24]$. The studies were conducted in the following geographic regions: one study was performed in Canada ( $n=462$ cases) [21], five in Europe ( $n=1,346$ cases) $[15,18,20,22,23]$, and two studies were performed in Asia $(\mathrm{n}=6,881$ cases $)[19,24]$.

\section{Methodological quality of studies}

We evaluated the methodological quality of studies included in the final analysis based on the NOS. The range of quality scores was 6 to 8 ; the average score was 6.0 for case-control studies and 7.8 for cohort studies. The high-quality studies (score of 8 in only cohort studies) included five cohort studies $[15,20,22,23,24]$.

\section{Use of hypnotics and the risk of or mortality from heart disease}

As reported in Fig. 2, use of hypnotics was significantly associated with a decreased risk of or mortality from heart disease in the random-effects meta-analysis of all eight studies (OR/RR, 0.84; 95\% CI, 0.79 to o.89). Table 2 exhibits results from subgroup meta-analyses by various factors. On analyzing by the type of heart disease, there was a significant relationship between the use of hypnotics and cardiovascular disease, but not in heart

495 Identified studies from the databases using keywords and bibliographies of relevant articles:

289 PubMed, 203 EMBASE, and 3 bibliographies

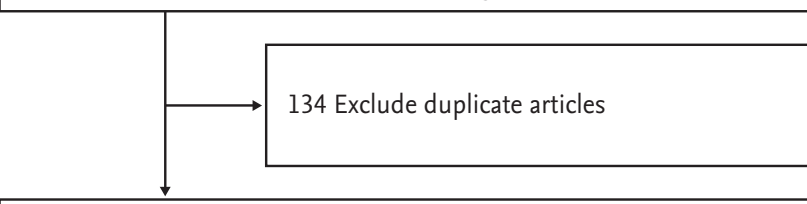

361 Articles remaining after excluding duplicates

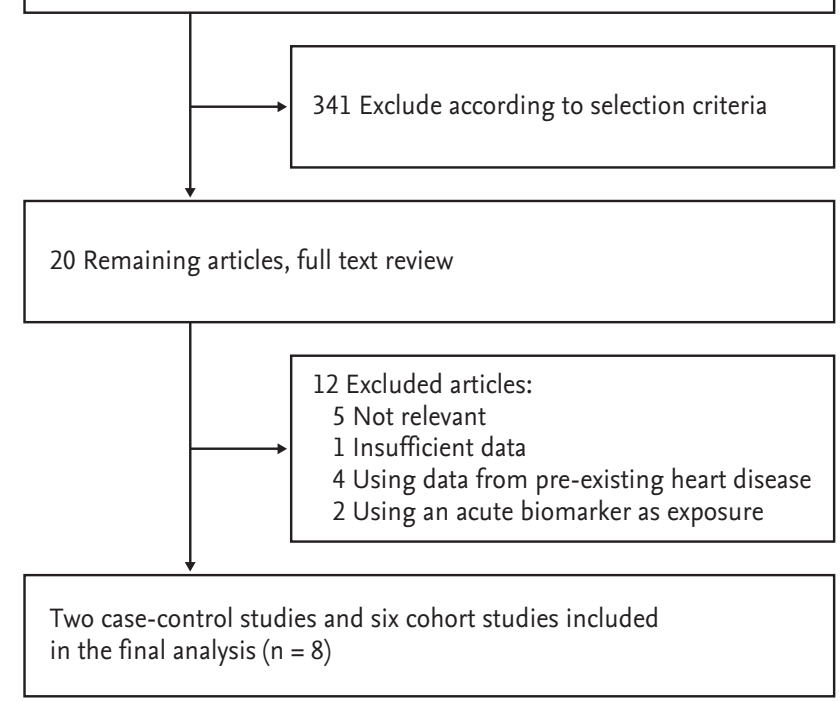

Figure 1. Flow diagram for identification of relevant studies. 


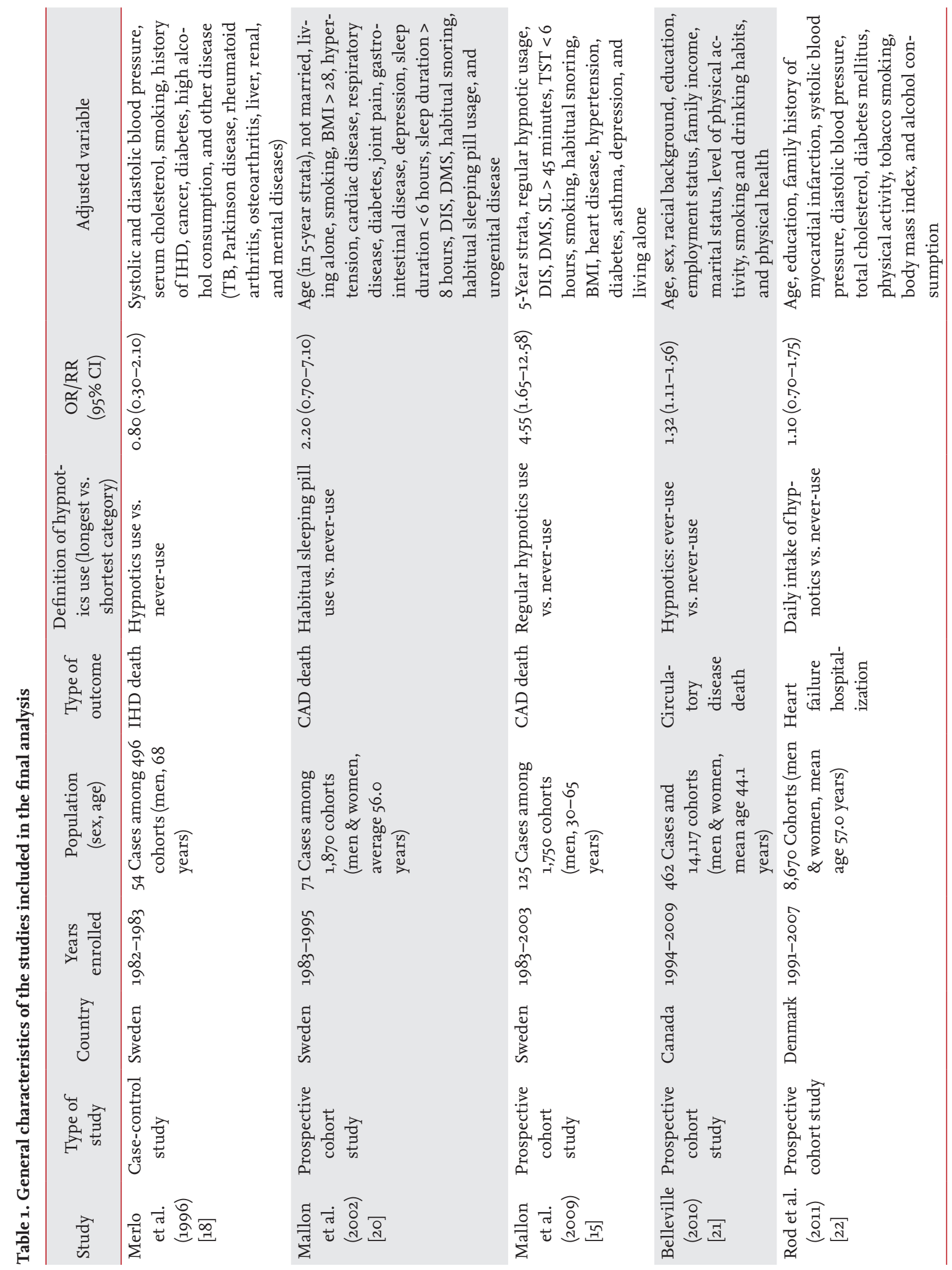




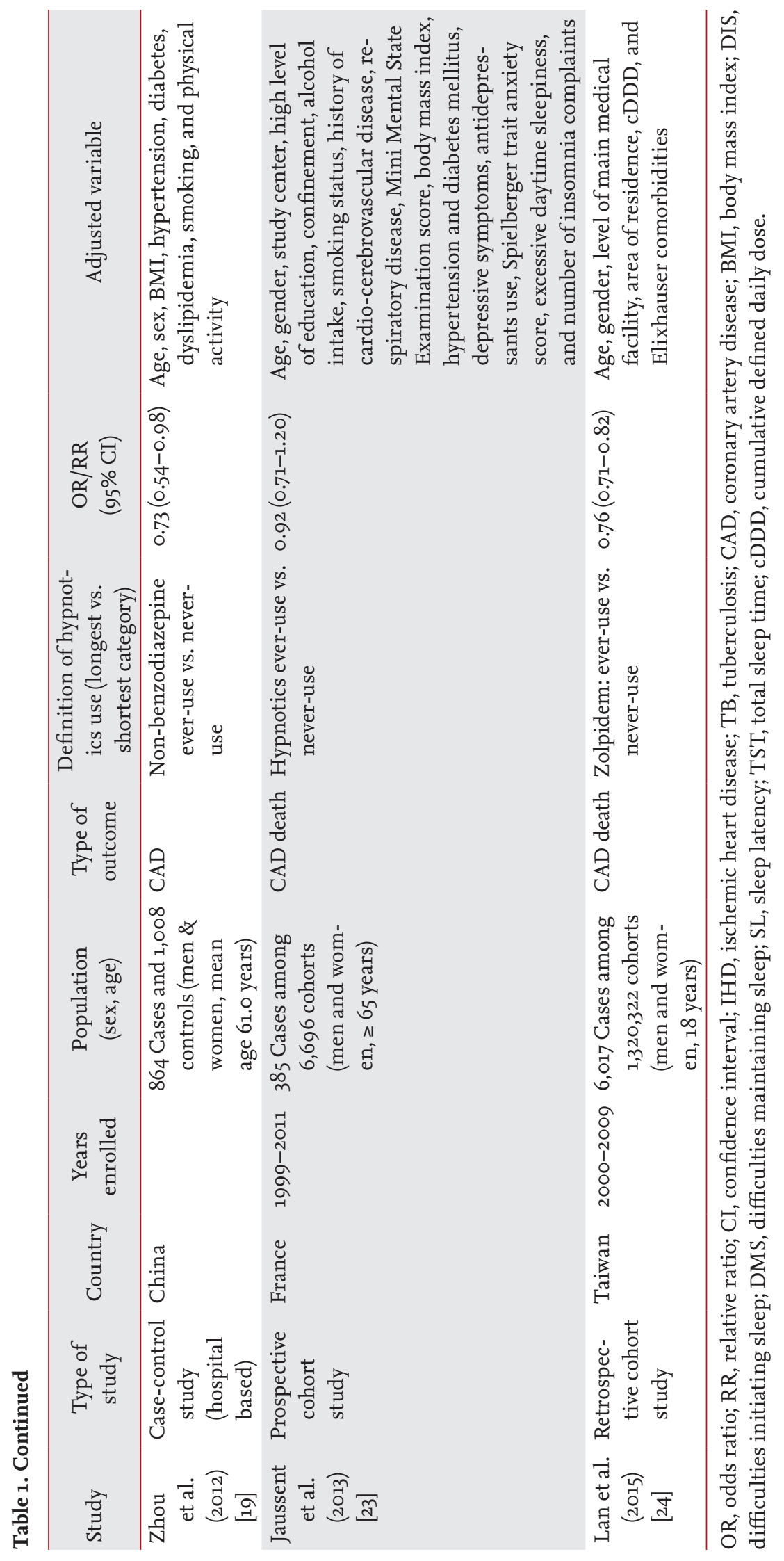




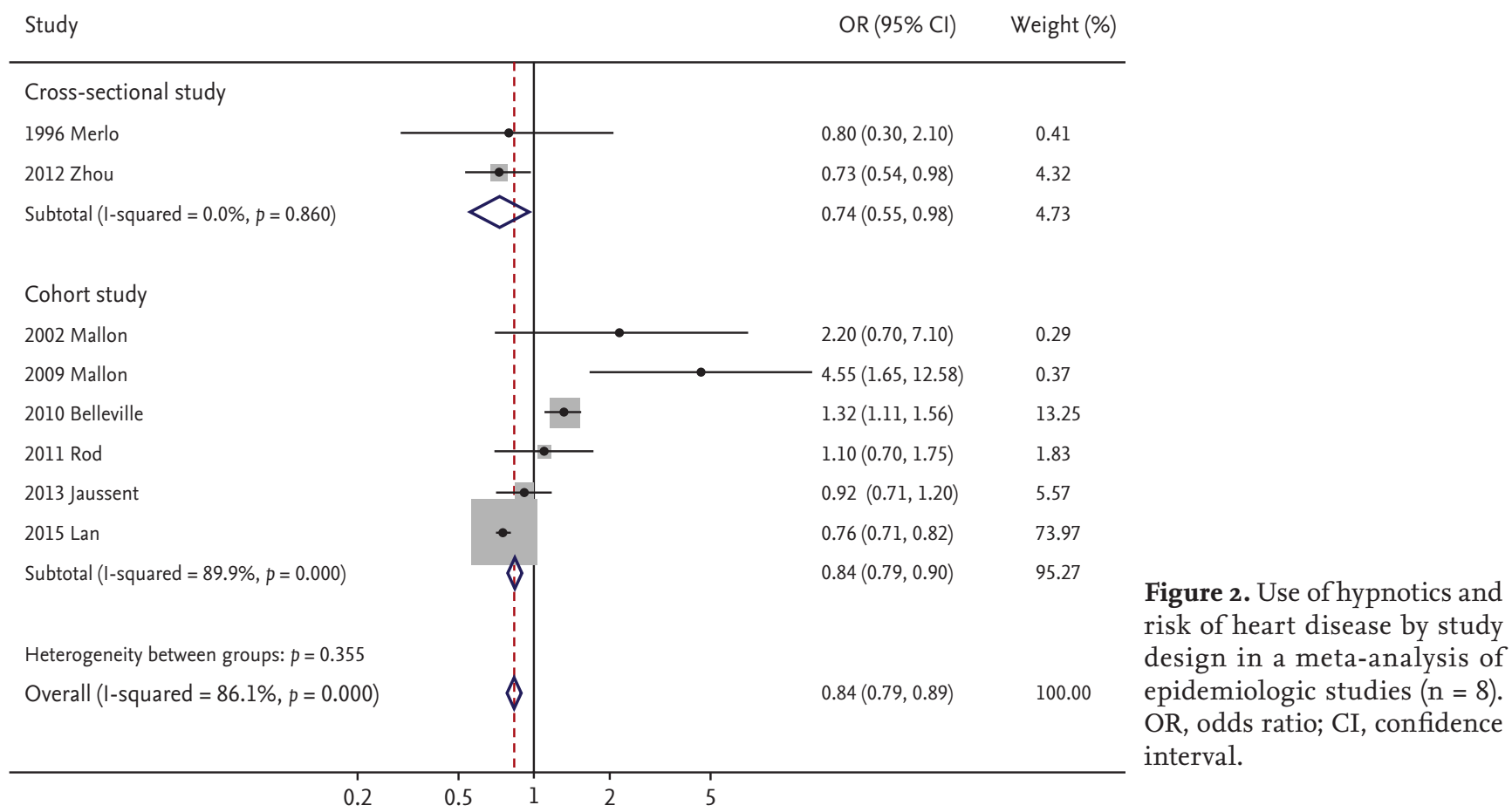

failure. Based on the severity of heart disease, intake of hypnotics was associated with mortality from heart disease but not with risk of heart disease. With respect to gender, studies including both male and female subjects reported the same association but not in studies which enrolled only male subjects. The same association remained significant in high-quality studies but not in low-quality studies. Larger studies showed a tendency towards a more elevated estimate of association in terms of a significant decreased risk of or mortality from cardiovascular outcomes, compared to smaller studies.

\section{Subgroup analysis based on the geographic region}

On stratifying by the geographic region, OR/RR was lowest in studies conducted in China/Taiwan compared to the studies conducted in Europe or Canada ( $p$ for studies conducted in Asia vs. non-Asian countries < 0.01) (Table 2). On dividing the geographic region into Western and Asian area, there was a positive relationship between the use of hypnotics and the risk of or mortality from heart disease in the Western area (pooled OR/RR, 1.21; 95\% CI, 1.06 to 1.38 with a significant heterogeneity; $I^{2}, 63.3 \%$ ).

\section{Type of hypnotics and risk of or mortality from heart disease}

The effects of hypnotics on risk of or mortality from heart disease were different according to the type of hypnotics (Table 2). Zolpidem users have a tendency for decreased risk of heart disease (OR/RR, 0.71; 95\% CI, 0.55 to 0.92$)$, but BZD use showed the possibility of increased risk of or mortality from heart disease (OR/RR, 1.80; 95\% CI, 1.77 to 1.84).

\section{Publication bias}

No publication bias was found in the selected studies (Begg's funnel plot was symmetric; Egger's test, $p$ for bias $=0.14$ ).

\section{DISCUSSION}

We found that the use of hypnotics was associated with a decreased risk of or mortality from heart disease in the current meta-analysis of observational epidemiological studies especially for Asia people.

There are several possible explanations for the association. First, previous systematic reviews of epidemiologic observational studies reported that insomnia was positively related with a risk of or mortality from cardiovascular disease [25]. In addition, there are emerging evidences that anxiety could be one of the important causes for the development of coronary artery disease and mor- 
Table 2. Association between hypnotics use and the risk of or mortality from heart disease in subgroup meta-analyses

\begin{tabular}{|c|c|c|c|c|}
\hline Factor & $\begin{array}{l}\text { No. of } \\
\text { studies }\end{array}$ & Summary OR/RR (95\% CI) & $\begin{array}{l}\text { Heterogeneity, } \\
\qquad I^{2}(\%)\end{array}$ & Model used \\
\hline All $[15,18-24]$ & 8 & $0.84(0.79-0.89)$ & 86.1 & Random-effects \\
\hline \multicolumn{5}{|l|}{ Type of heart disease } \\
\hline Cardiovascular disease $[15,18-21,23,24]$ & 7 & $0.84(0.78-0.89)$ & 87.8 & Random-effects \\
\hline Heart failure [22] & 1 & $1.10(0.70-1.75)$ & NA & NA \\
\hline \multicolumn{5}{|l|}{ Severity of heart disease } \\
\hline Risk $[19,22]$ & 2 & $0.82(0.64-1.06)$ & $53 \cdot 7$ & Random-effects \\
\hline Mortality $[15,18,20,21,23,24]$ & 6 & $0.84(0.79-0.90)$ & 89.6 & Random-effects \\
\hline \multicolumn{5}{|l|}{ Sex } \\
\hline Male only $[15,18]$ & 2 & $1.84(0.91-3.71)$ & 83.0 & Random-effects \\
\hline Male \& female [19-24] & 6 & $0.84(0.78-0.89)$ & 87.4 & Random-effects \\
\hline \multicolumn{5}{|l|}{ Region } \\
\hline America $[21]$ & 1 & $1.32(1.11-1.56)$ & NA & NA \\
\hline Europe $[15,18,20,22,23]$ & 5 & $1.05(0.85-1.30)$ & 63.3 & Random-effects \\
\hline Western $[15,18,20-23]$ & 6 & $1.21(1.06-1.38)$ & 63.3 & Random-effects \\
\hline Asia $[19,24]$ & 2 & $0.76(0.71-0.81)$ & o & Fixed-effect \\
\hline \multicolumn{5}{|l|}{ Duration of zolpidem use, yr } \\
\hline $0-5$ & 1 & $0.91(0.63-1.32)$ & NA & NA \\
\hline $5^{-10}$ & 1 & $0.64(0.38-0.97)$ & NA & NA \\
\hline$>10$ & 1 & $0.42(0.18-0.85)$ & NA & NA \\
\hline \multicolumn{5}{|l|}{ Type of hypnotics } \\
\hline Zolpidem $[19,23]$ & 2 & $0.71(0.55-0.92)$ & 0 & Fixed-effect \\
\hline Benzodiazepine $[19,23,24]$ & 3 & $1.80(1.77-1.84)$ & 88.3 & Random-effects \\
\hline \multicolumn{5}{|l|}{ Case-control study design } \\
\hline Population-based $[15,18,20-24]$ & 7 & $0.84(0.79-0.90)$ & 87.9 & Random-effects \\
\hline Hospital-based [19] & 1 & $0.73(0.54-0.98)$ & NA & NA \\
\hline \multicolumn{5}{|l|}{ Methodological quality } \\
\hline High quality $[15,20,22-24]$ & 5 & $0.79(0.73-0.84)$ & 78.7 & Random-effects \\
\hline Low quality $[18,19,21]$ & 3 & $1.13(0.98-1.31)$ & 83.3 & Random-effects \\
\hline \multicolumn{5}{|l|}{ Sample size } \\
\hline$<5,067$ subjects $[15,18,20,23]$ & 4 & $0.88(0.68-1.16)$ & 78.6 & Random-effects \\
\hline$>5,068$ subjects $[19,21,22,24]$ & 4 & $0.84(0.78-0.89)$ & 91.7 & Random-effects \\
\hline
\end{tabular}

OR, odds ratio; RR, risk ratio; CI, confidence interval; NA, not applicable.

tality from cardiac diseases $[26,27]$. In an animal study in which zopiclone or zolpidem were administrated to rat, zopiclone exhibited a lowering effect on anxiety behavior [28]. Therefore, it is possible that hypnotics can lower the risk of coronary artery disease by alleviating insomnia or anxiety.

Second, as a considerable part of incidence of and/ or mortality from heart disease can be attributable to many confounding factors such as smoking, alcohol, hypertension, diabetes, dyslipidemia, obesity, physical inactivity, and family history of heart disease, they could underlie the relationship [29-31]. In this meta-analysis, seven studies [15,18-23] included smoking status as a confounding factor, and the significant negative association disappeared (OR/RR, 1.11; 95\% CI, 0.98 to 1.25). And there can be another undetected confounding factors which may contribute to the association between the use of hypnotics and risk of heart disease. 
Our meta-analysis showed that the BZDs can increase the risk of or mortality from heart diseases unlike non-BZDs. Our finding is coherent with the result in the study by Lapane et al. [32] in which ischemic heart disease occurrence was two times in BZD users than non-users. The possible detrimental effects of BZD on heart disease also can be seen at a laboratory study which looked at the effects of BZD on heart rate. Use of triazolam or flurazepam as hypnotics resulted in a significant elevation of heart rate that was existing for up to 4 hours during sleep [33]. Hypotension can often be observed in subjects who overuse BZDs than average [34]. One possible mechanism by which BZD intake can be connected to the risk of heart disease is infection with virus. When a total of 1,682 people who had no HIV at baseline were followed for about 80 months [35], BZD use was significantly associated with an increased rate of HIV seroconversion (adjusted risk ratio, 1.50; 95\% CI, 1.01 to 2.24). A cross-sectional study enrolling a total 354 subjects [36] showed that cardiovascular disease occurrence was higher in HIV-infected patients than HIV-negative subjects. Another virus which can connect use of hypnotics and the risk of heart disease can be hepatitis $\mathrm{C}$ virus (HCV). In a prospective study of the AIDS Care Cohort and Vancouver Injection Drug Users Study (VIDUS) cohorts followed-up from 1996 to 2013 in Canada [37], there was a significant relationship between BZD use and seroconversion of HCV. Chronic infection with $\mathrm{HCV}$ can play a pivotal role for growing prevalence for ischemic heart disease, independent of the conventional risk factors for cardiovascular events or the severity of liver disease [38].

We found that there was a racial difference in the effect of hypnotics on risk of or mortality from heart disease. In a report from the Multiethnic Study of Atherosclerosis (MESA) [39], the prevalence of calcification measured by coronary calcium score in computed tomography was $70 \%$ in white men, $59 \%$ in Chinese men after adjusting conventional risk factors. Japanese also had higher HDL cholesterol levels which is related with decreased risk of cardiovascular disease than whites [40]. The frequency of the $\mathrm{T}$ allele for hepatic lipase which is associated with higher level of HDL cholesterol as well as lower level of triglycerides was three times higher in Japanese than in Caucasians. The defensive cardiovascular mechanisms of hypnotics especially observed in Asian is not well known and need additional explorations.

Our meta-analysis has several strengths. We investigated a large number of subjects with a long time of follow-up. All of included studies were population-based with the exception of the study by Zhou et al. [19]. This gives a high statistical power for examining the relationship between hypnotics use and risk of heart disease. Another advantage of our study is that no significant publication bias was observed.

Our study has several limitations. First, we only included observational epidemiological studies because few randomized controlled trials have been published on this topic so far. In general, case-control studies are more susceptible to biases, such as selection bias and recall bias than cohort studies. In addition, cohort studies have a lower level of evidence than randomized controlled trials. Thus, our meta-analysis does not provide the high level of evidence. Second, definitions of use of hypnotics in each study were fairly heterogeneous. We used the highest and lowest levels of hypnotics use about the exposure definition in most studies. This could have led to heterogeneity. Third, most of data were acquired from questionnaires; hence, the possibility of encountering bias due to misinterpretation of the question can exist. Finally, we found significant heterogeneity across studies, which may have resulted from differences in study designs, sample sizes, heart disease measures, analysis strategies, and participants' characteristics.

\section{KEY MESSAGE}

1. The association between hypnotics intake and the risk of heart disease remains controversial in several observational epidemiological studies.

2. We investigated the associations by performing a meta-analysis.

3. Our meta-analysis of observational epidemiological studies found that use of hypnotics was associated with a decreased risk of or mortality from heart disease.

\section{Conflict of interest}

No potential conflict of interest relevant to this article was reported. 


\section{REFERENCES}

1. Colten HR, Altevogt BM. Sleep Disorders and Sleep Deprivation: An Unmet Public Health Problem. Washington (DC): National Academies Press, 2006.

2. Nomura K, Yamaoka K, Nakao M, Yano E. Impact of insomnia on individual health dissatisfaction in Japan, South Korea, and Taiwan. Sleep 2005;28:1328-1332.

3. Ancoli-Israel S, Roth T. Characteristics of insomnia in the United States: results of the 1991 National Sleep Foundation Survey. I. Sleep 1999;22 Suppl 2:S347-S353.

4. Morin CM, LeBlanc M, Daley M, Gregoire JP, Merette C. Epidemiology of insomnia: prevalence, self-help treatments, consultations, and determinants of help-seeking behaviors. Sleep Med 2006;7:123-130.

5. Mellinger GD, Balter MB, Uhlenhuth EH. Insomnia and its treatment: prevalence and correlates. Arch Gen Psychiatry 1985;42:225-232.

6. Ohayon MM, Caulet M, Priest RG, Guilleminault C. Psychotropic medication consumption patterns in the UK general population. J Clin Epidemiol 1998;51:273-283.

7. Vermeeren A. Residual effects of hypnotics: epidemiology and clinical implications. CNS Drugs 2004;18:297-328.

8. Alvarenga JM, Loyola Filho AI, Firmo JO, Lima-Costa MF, Uchoa E. Prevalence and sociodemographic characteristics associated with benzodiazepines use among community dwelling older adults: the Bambui Health and Aging Study (BHAS). Rev Bras Psiquiatr 2008;30:7-11.

9. Sanna E, Busonero F, Talani G, et al. Comparison of the effects of zaleplon, zolpidem, and triazolam at various GABA(A) receptor subtypes. Eur J Pharmacol 2002;451:103110.

10. Inagaki T, Miyaoka T, Tsuji S, Inami Y, Nishida A, Horiguchi J. Adverse reactions to zolpidem: case reports and a review of the literature. Prim Care Companion J Clin Psychiatry 2010;12:e1-e8.

11. Mailliet F, Galloux P, Poisson D. Comparative effects of melatonin, zolpidem and diazepam on sleep, body temperature, blood pressure and heart rate measured by radiotelemetry in Wistar rats. Psychopharmacology (Berl) 2001;156:417-426.

12. Jehle J, Ficker E, Wan X, et al. Mechanisms of zolpideminduced long QT syndrome: acute inhibition of recombinant hERG $\mathrm{K}(+)$ channels and action potential prolongation in human cardiomyocytes derived from induced pluripotent stem cells. Br J Pharmacol 2013;168:1215-1229.
13. Kim DH, Lee JT, Lee IK, Ha JH. Comparative anticancer effects of flavonoids and diazepam in cultured cancer cells. Biol Pharm Bull 2008;31:255-259.

14. Weissinger J. NDA 19-908 ambien pharmacology memos \& exclusivity summary [Internet]. Silver Spring (US): U.S. Food and Drug Administration, c1992 [cited 2017 Jul 21]. Available from: http://www.accessdata.fda.gov/drugsatfda_ docs/nda/pre96/019908_Sooo_PHARM_MEMOS\&EX CLUSIVITY_SUMMARY.pdf.

15. Mallon L, Broman JE, Hetta J. Is usage of hypnotics associated with mortality? Sleep Med 2009;10:279-286.

16. Higgins JP, Thompson SG. Quantifying heterogeneity in a meta-analysis. Stat Med 2002;21:1539-1558.

17. DerSimonian R, Laird N. Meta-analysis in clinical trials. Control Clin Trials 1986;7:177-188.

18. Merlo J, Hedblad B, Ogren M, et al. Increased risk of ischaemic heart disease mortality in elderly men using anxiolytics-hypnotics and analgesics: results of the 10year follow-up of the prospective population study "Men born in 1914", Malmo, Sweden. Eur J Clin Pharmacol 1996;49:261-265.

19. Zhou X, Zhang Y, Chen J, Xu W. Use of non-benzodiazepine hypnotics is associated with decreased risk of coronary artery disease. Intern Med 2012;51:829-832.

20. Mallon L, Broman JE, Hetta J. Sleep complaints predict coronary artery disease mortality in males: a 12-year follow-up study of a middle-aged Swedish population. J Intern Med 2002;251:207-216.

21. Belleville G. Mortality hazard associated with anxiolytic and hypnotic drug use in the National Population Health Survey. Can J Psychiatry 2010;55:558-567.

22. Rod NH, Andersen I, Prescott E. Psychosocial risk factors and heart failure hospitalization: a prospective cohort study. Am J Epidemiol 2011;174:672-680.

23. Jaussent I, Ancelin ML, Berr C, et al. Hypnotics and mortality in an elderly general population: a 12-year prospective study. BMC Med 2013;11:212.

24. Lan TY, Zeng YF, Tang GJ, et al. The use of hypnotics and mortality: a population-based retrospective cohort study. PLoS One 2015;10:e0145271.

25. Sofi F, Cesari F, Casini A, Macchi C, Abbate R, Gensini GF. Insomnia and risk of cardiovascular disease: a meta-analysis. Eur J Prev Cardiol 2014;21:57-64.

26. Vogelzangs N, Seldenrijk A, Beekman AT, van Hout HP, de Jonge P, Penninx BW. Cardiovascular disease in persons with depressive and anxiety disorders. J Affect Dis- 
ord 2010;125:241-248.

27. Roest AM, Martens EJ, de Jonge P, Denollet J. Anxiety and risk of incident coronary heart disease: a meta-analysis. J Am Coll Cardiol 2010;56:38-46.

28. Huang MP, Radadia K, Macone BW, Auerbach SH, Datta S. Effects of eszopiclone and zolpidem on sleep-wake behavior, anxiety-like behavior and contextual memory in rats. Behav Brain Res 2010;210:54-66.

29. Price JF, Fowkes FG. Risk factors and the sex differential in coronary artery disease. Epidemiology 1997;8:584-591.

30. Gensini GF, Comeglio M, Colella A. Classical risk factors and emerging elements in the risk profile for coronary artery disease. Eur Heart J 1998;19 Suppl A:A53-A61.

31. Virmani R, Farb A, Burke AP. Risk factors in the pathogenesis of coronary artery disease. Compr Ther 1998;24:519-529.

32. Lapane KL, Zierler S, Lasater TM, Barbour MM, Carleton $\mathrm{R}$, Hume AL. Is the use of psychotropic drugs associated with increased risk of ischemic heart disease? Epidemiology 1995;6:376-381.

33. Muzet A, Johnson LC, Spinweber CL. Benzodiazepine hypnotics increase heart rate during sleep. Sleep 1982;5:256-
261.

34. Hojer J. Management of benzodiazepine overdose. CNS Drugs 1994;2:7-17.

35. Ickowicz S, Hayashi K, Dong H, et al. Benzodiazepine use as an independent risk factor for HIV infection in a Canadian setting. Drug Alcohol Depend 2015;155:190-194.

36. Kingery JR, Alfred Y, Smart LR, et al. Short-term and long-term cardiovascular risk, metabolic syndrome and HIV in Tanzania. Heart 2016;102:1200-1205.

37. Bach P, Walton G, Hayashi K, et al. Benzodiazepine use and hepatitis $\mathrm{C}$ seroconversion in a cohort of persons who inject drugs. Am J Public Health 2016;106:1067-1072.

38. Domont F, Cacoub P. Chronic hepatitis C virus infection, a new cardiovascular risk factor? Liver Int 2016;36:621-627.

39. Bild DE, Detrano R, Peterson D, et al. Ethnic differences in coronary calcification: the Multi-Ethnic Study of Atherosclerosis (MESA). Circulation 2005;111:1313-1320.

40. deLemos AS, Wolfe ML, Long CJ, Sivapackianathan R, Rader DJ. Identification of genetic variants in endothelial lipase in persons with elevated high-density lipoprotein cholesterol. Circulation 2002;106:1321-1326. 